\title{
A Platform for Solving the Ecologist's Dilemma; Setting Conservation Procedures
}

\author{
Nicole Benjamin-Fink ${ }^{1 *}$ and Brian K Reilly ${ }^{2}$ \\ ${ }^{1}$ Conservation Beyond Borders, Director's Desk, USA \\ ${ }^{2}$ Department of Nature Conservation, Tshwane University of Technology, South Africa
}

*Corresponding author: Nicole Benjamin-Fink, Conservation Beyond Borders, Director's Desk, 3033 Excelsior Blvd, \#575, 55416, Minnesota, USA.

Received Date: November 18, 2019

Published Date: November 27, 2019

\begin{abstract}
The ecologist's dilemma is worldwide and straightforward; providing wildlife management recommendations, while constrained by scarce resources and limited data. We apply Object Oriented Bayesian Networks (OOBNs) to quantify complexities within their ecological context. We generate and utilize expert understanding of uncertainties. Key variables are identified and clustered within spatial, biological, and market domains. Specifically, (i) blue wildebeest male to black wildebeest male ratio, and (ii) spatial connectivity.

We put forth two OOBNs. Varying in their scope, each accurately represents the wicked problem concerning wildebeest hybridization and addresses different objectives faced by decision-makers. A detailed OOBN quantifies spillover implications, while a skeleton 0OBN assesses associated risks. We equip ecologist with effective tools to resume informed decisions and maximize resources. Lastly, we offer a decision tree that promotes the usage of OOBNs as user friendly tools aimed at solving for the ecologist's dilemma while addressing similar ecological and environmental uncertainties worldwide.
\end{abstract}

Keywords: Applied resources management; Ecologist's dilemma; Hybridization; Object-oriented-bayesian-network; Policy making; Wicked problems; Wildebeest

\section{Introduction}

\section{The challenge in "Wicked” Problems}

Researchers are increasingly faced with 'Wicked' problems; multifaceted complex challenges that span disciplines, knowledge bases, and value systems [1]. They are sequential to other problems, lack clear solution, and may be easily misrepresented as an isolated concern [2]. Ecological and environmental wicked challenges are often rooted in spillover effects from biodiversity, ecosystem services, social and environmental aspects, management implications, and economic profitability goals [3-6].

\section{The ecologist's decision-maker's dilemma}

We coin the phrase 'The ecologist's dilemma'. On the one horn, ecologists are task with providing wildlife managers and environmental agencies with guidelines aimed at managing populations. On the other hand, scarce data and limited resources pose significant constraints. More often than not, the complexity of this dilemma is intensified by multidisciplinary linkages, unpredictability, and competing stakeholder objectives. Consequentially, many current paradigms leverage existing best practices (i.e., "grandfathered-in" solutions) [7]. However, this may result in "Type III error" whereby an effective solution is employed to address the wrong problem [8].

A precise understanding of key and embedded variables is required for an effective and relevant solution $[9,10]$. However, traditional liner modelling may be ineffective in the face of imperfect information [11]. There is a need to employ an innovative approach which accounts for tradeoffs with the purpose to standardize the procedure of ecology-based decision-making. Solving for the ecological decision-makers' dilemma will help to advance policy and resource management implications worldwide. A noble approach should be multi-disciplinary and recognize spillover effects of wildlife management decisions into other domains, user friendly, and capable of accounting for all aspects of the overarching wicked problem. 


\section{The wicked case Study of hybridization}

Hybridization is one of the primary wicked problems of our time [4]. Accelerating at an alarming rate, it is responsible for the genetic extinction of endangered and indigenous species worldwide (Box 1). Here, we demonstrate the applicability of Object-Oriented Bayesian Networks (OOBNs) to solve for the ecologist's dilemma (i.e., constraints posed by limited data and scares resources). This platform for standardizing decision making is presented within the contextual framework of the case study concerning hybridization between the endemic black wildebeest (Connochaetes gnou) and the more common blue wildebeest (Connochaetes taurinus) in South Africa.

To date, genetic markers that differentiate pure breed wildebeest individuals from varying generations of hybrids have not yet been identified, and qualitative data are imprecise [12-14]. Ecologists are forced to provide guidelines for wildlife populations and landscapes management with economic profitability in mind, and under limited resources. Although such decisions are made on an ongoing basis, they are often "grandfathered in" [15] cautions that "grandfathered in" practices (which address inbreeding and outbreeding concerns) should be rigorously evaluated and adapted, considering hybridization concerns.

\section{BNs are underutilized in conservation research due to computational expense}

Bayesian Networks (BNs) are one of the most useful tools within the probabilistic knowledge representation and reasoning discipline applicable to modelling for reliability, dependency, risk analysis, diagnosis, and monitoring [16-18]. Recently, BNs have been used to model diverse ecology-based problems of high complexity, mainly concerning water, fisheries management, crop disease, and conservation [19]. However, during 1990-2010 a merely $4.2 \%$ of peer reviewed research, related to the environmental field utilized them, suggesting that this predictive tool remains unexploited [20].

BNs' underutilization is primarily attributed to associated computational expense and a gradual learning curve $[3,11]$. Deriving a BNs structure and populating it with expert knowledge is proven to be difficult and time consuming [21].

Despite their underutilization, BNs are a useful tool for addressing the wicked nature of ecology and environmental research [22]. Specifically, with bidirectional flow enables ecologists to populate networks with data as it becomes available; thus, serving as a tool that optimizes informed decisions given scarce, and seemingly insufficient, data. Here, we provide a platform for setting conservation standards to solve for the ecologist's dilemma, and thereby promote informed decision making and aid resource management in similar situations worldwide.

\section{Goal}

We address the long-standing ecologist's dilemma. We put forth two frameworks that informed decision in the face of scarce data and limited resources. We illustrate the applicability of Bayesian modeling and inference within the ecological and environmental discipline by solving for the case study of hybridization between black wildebeest and blue wildebeest.

\section{Methods}

\section{Study area}

Knowledge acquisition was generated from key experts concerning drivers for black wildebeest and blue wildebeest in the following five provinces: Limpopo, Gauteng, Mpumalanga, KwaZulu Natal, and The Free State (Map 1).

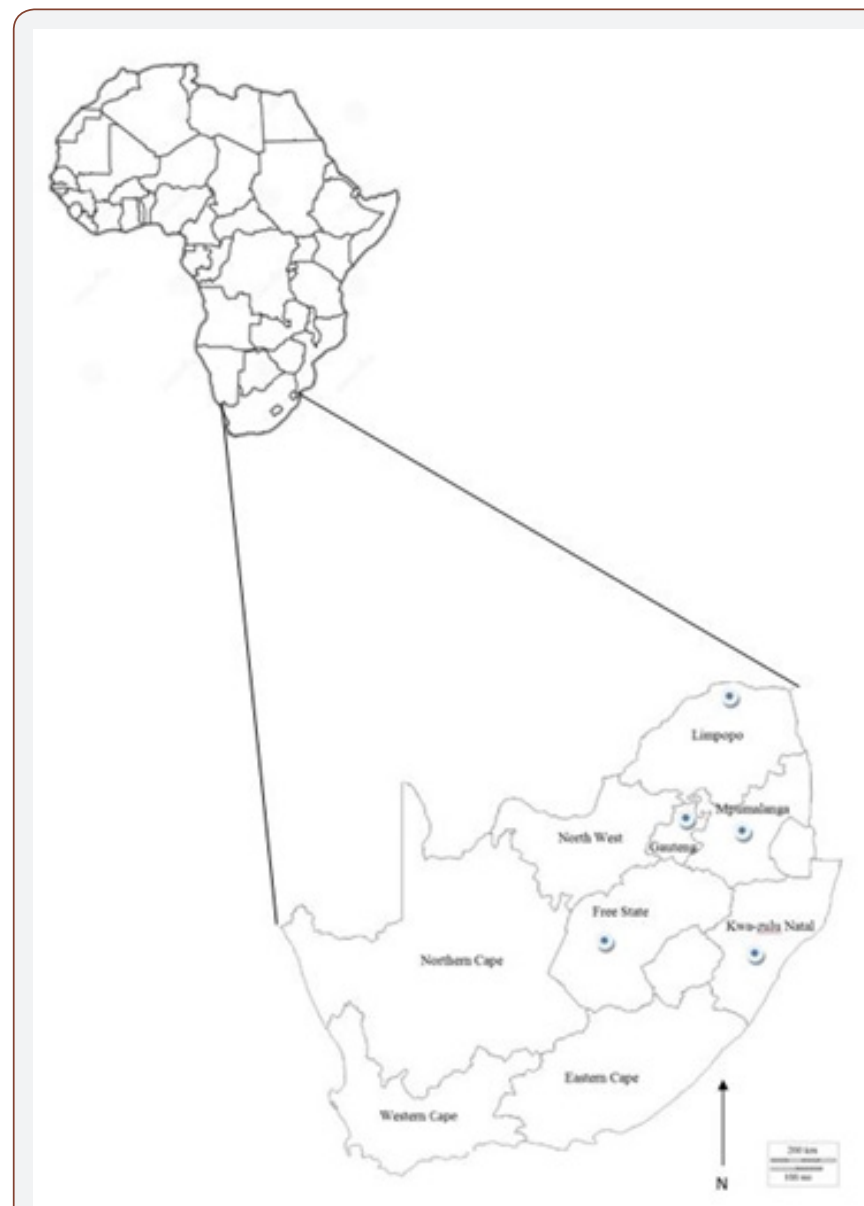

Map 1: Map of South African provinces. Surveyed locations are illustrated by ovals and within the following provinces: Limpopo, Gauteng, Mpumalanga, Kwazulu-Natal, and The Free State.

\section{Software}

We used the software Netica [23]. Once the programmer defines the model structure, Netica utilizes EM algorithms to construct conditional probabilities accounting for confidence levels of stakeholders in their ability to accurately predict probabilistic reasoning [24].

\section{Knowledge utilization}

There is a need for standardizing the procedure of knowledge utilization in the face of imperfect information and limited resources. The process of such informed decision making is via a platform that utilizes knowledge acquisition knowledge utilization consisted of two sources: peer reviewed literature, and 
expert understanding (often referred to as expert opinion, expert knowledge, or actionable data) (Figure 1).

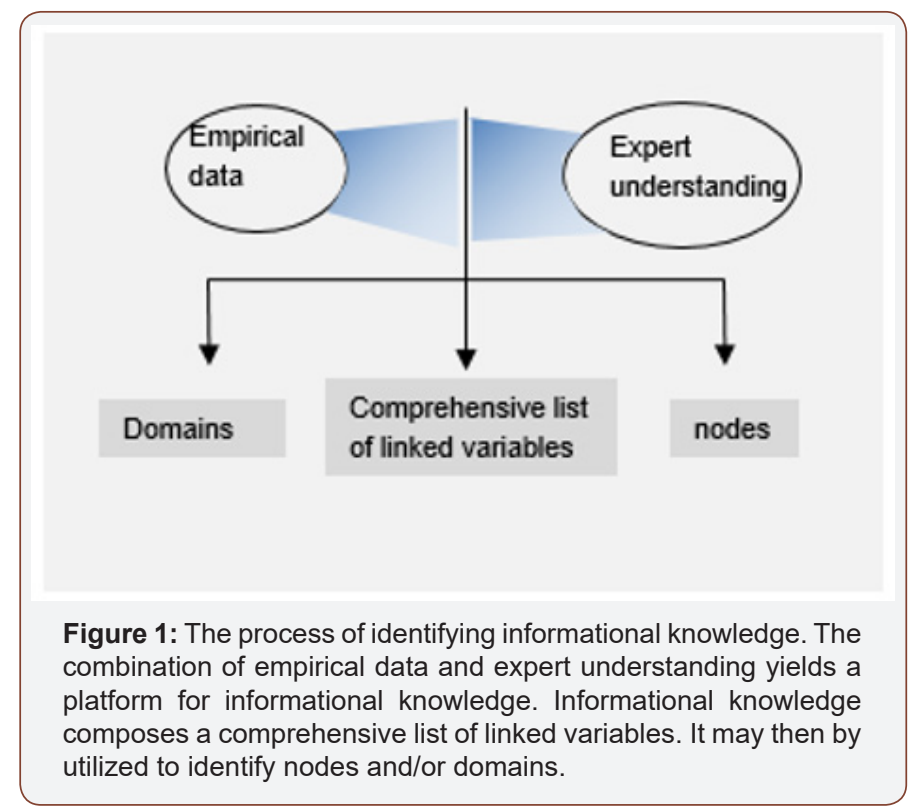

Figure 1 The process of identifying informational knowledge. The combination of empirical data and expert understanding yields a platform for informational knowledge. Informational knowledge composes a comprehensive list of linked variables. It may then by utilized to identify nodes and/or domains.

Literature review: A comprehensive list of variables linked to wildebeest biology and ecological requirements was formed following a literature review (Fig. 1). Specifically, search words included the following: wildebeest, wildebeest ecology, hybridization, hybrid zones.

\section{Expert understanding:}

a. Identify Key Stakeholder: Stakeholders are considered experts from a range of field. These include private ranch owners, managers, scientists, and governmental officials. They are users of knowledge and resources and are responsible for decision-making process and ultimately, adopt and enforce management plans. We additionally documented their seemingly competitive objectives.

b. Knowledge generation: The fundamental principle of inference and induction is to predict future events by utilizing past data. We generated useable expert knowledge by asking experts the following three questions: (1) identify variables (positively or negatively) linked to the process of hybridization, (2) generate realistic probabilities (i.e., 0-1) for each node (i.e., if the variable were to occur 100 times, how many times would it be linked to hybridization), and (3) predict posterior consequences and provide reasoning. Segments of knowledge were additionally used to verify, re-evaluate, and recalibrate overall OOBNs for inaccuracies. We used the widest appropriate for a priori data so that risks and management tradeoffs could be identified and prioritized.

\section{Model variable and structure elicitation}

We treated BNs as subnetworks for an interlinking hierarchical OOBN. The process of variable and structure elicitation is artificially divided (Figure 2). User validation is embedded in both these phases.

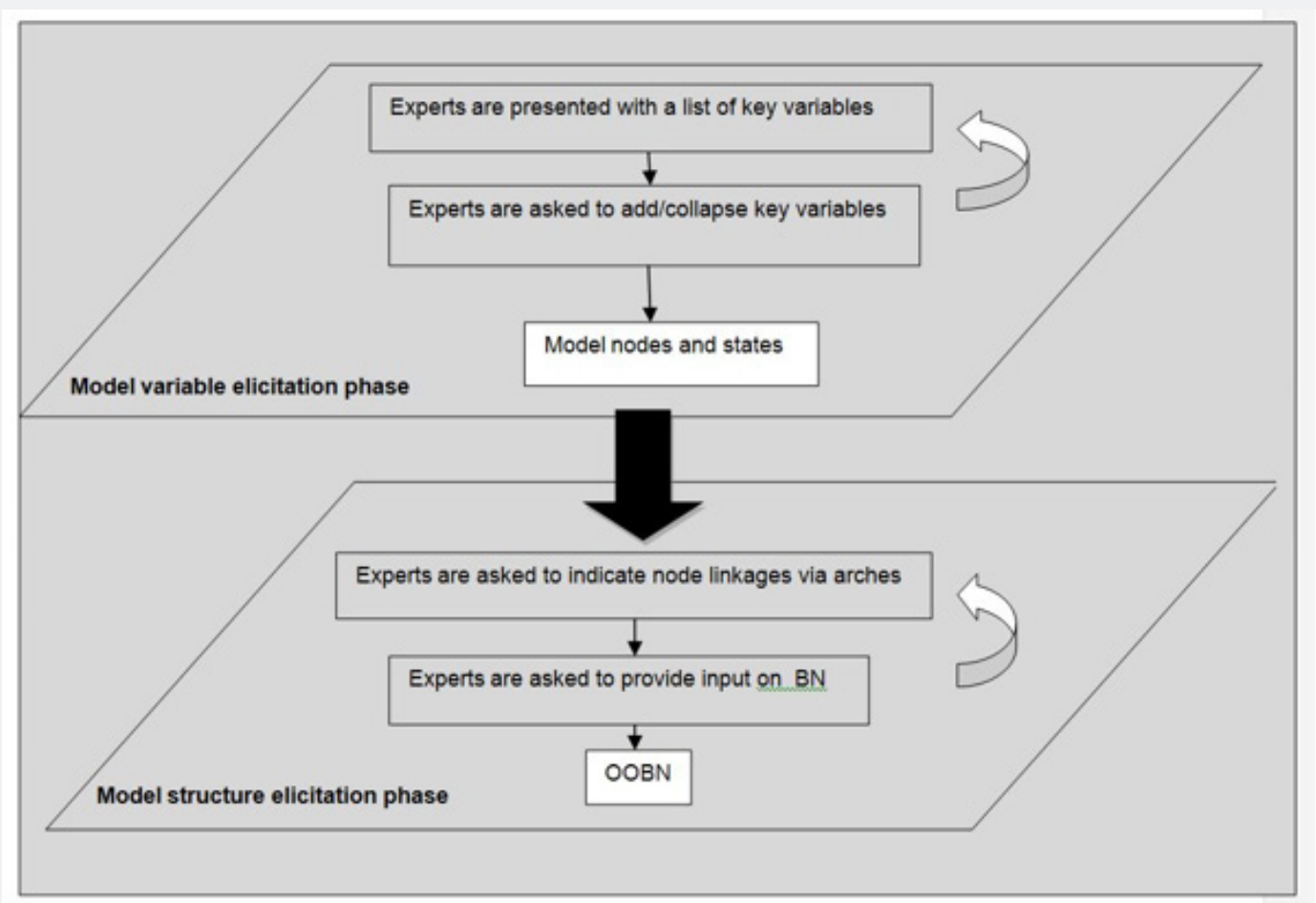

Figure 2: Model variable and structure elicitation for target node. Target nodes are specific to the ecological phenomenon at hand. Phases are outlined in layers. Processes are listed in gray boxes and products are listed in white boxes. White arrows indicate repetitiveness. Black narrow arrows indicate sequential steps within layers. 
Parameterization: The fundamental principle of inference and induction is to predict future (posterior probabilities) events by utilizing past data (a priori data). The effective generation of appropriate and useable knowledge was derived during a workshop by asking experts the following: (1) identify and rate factors contributing, facilitating, or countering the process of hybridization; (2) generate the widest possible range of realistic probabilities for each node, (3) predict posterior consequences and provide reasoning; and (4) determine feasibility and efficiency of various management practices. Parameters contain knowledge and descriptions of its manipulation of the knowledge, allowing them to have a state and quantifiable or categorical behavior (i.e., an identifiable role) (Figure 2).

Domain identification: A domain is a cluster of parameters that share a commonality in structure or behavior, which provides a working interface for the programmer to interact with clusters of moving parts that differ in their predictability of hybridization. This additionally enables replication in similar ecological systems.

Validation: We sought to determine via "user validation" whether the information generated might be altered by the structure of the model. Segments of expert knowledge were used to verify, re-evaluate, and recalibrate BNs, and OOBNs for inaccuracies. Deviations from predictions would have indicated errors in the network structure.

\section{Results}

\section{Utilization of expert knowledge conceptualizes ecological complexities via abstract visualization}

Experts are users of knowledge and resources, responsible for decision-making process, and ultimately, adopt and enforce management plans. Their seemingly competitive objectives and needed tools were documented. Although their primary objectives differ, they all agree that the ecologists provide critical guidelines for decisions (Box 2). Moreover, they differ in their need and usage of tools, that would otherwise assist in informed decisions and resource maximization.

OOBNs illuminate linkages among variables which otherwise may seemingly be ignored due to isolation analysis (Figure 3). The objectives of the abstract visualization are threefold: (1) to promote an understanding of which factors require attention when managing for hybridization occurrences, (2) to identify decision maker's scope of focus, and (3) to identify spillover effects. Parameters were clustered into three: market, biological, and spatial (Figure 3).

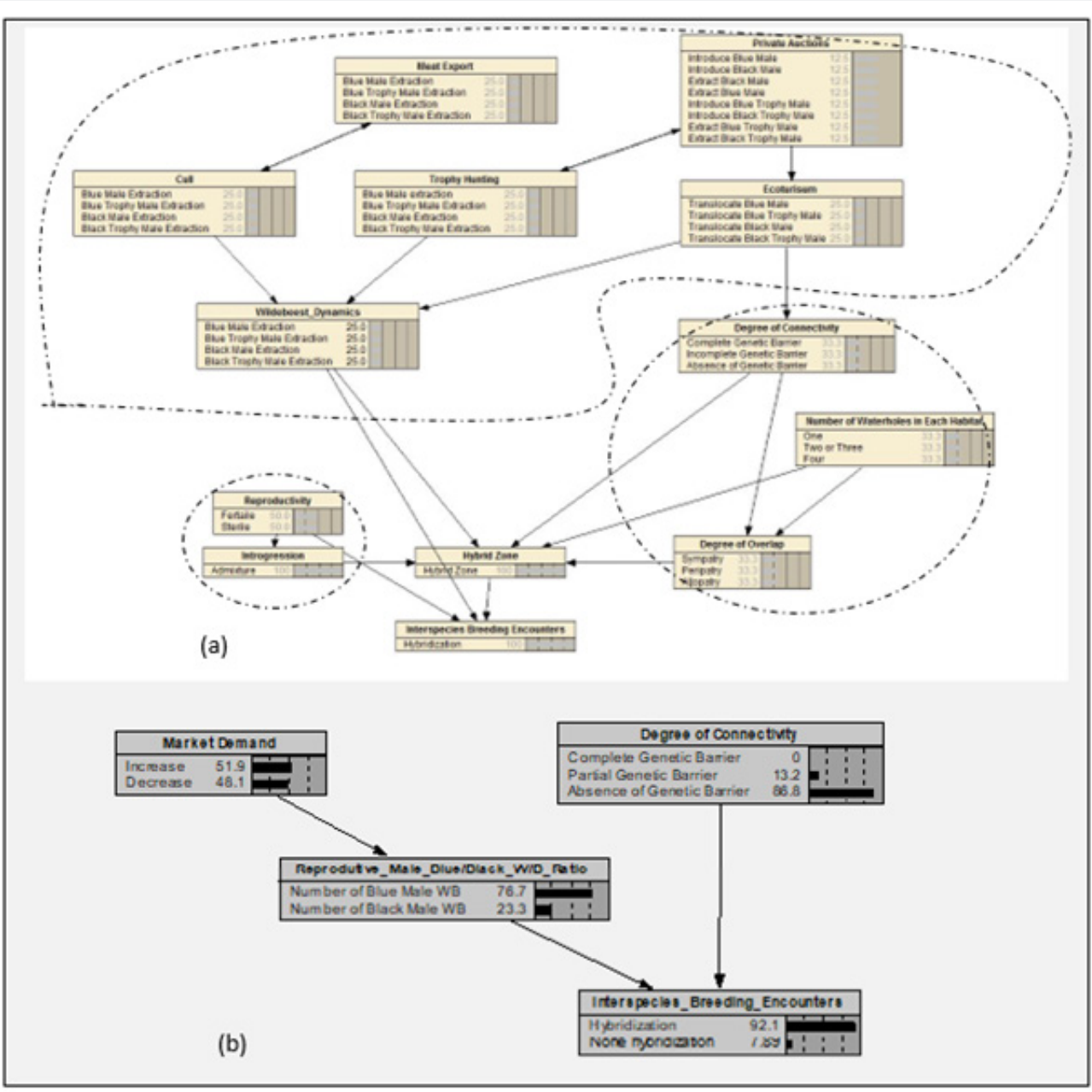

Figure 3: Domains are outlined in dotted circumferences. OOBN differ in their goal; (a) provides ecologists with a detail quantification of spillover effects, (b) provides ecologists with an "eagle's-eye" perspective, acting as a risk assessment tool for resource allocation maximization. Both models may be utilized sequentially or in isolation. 


\section{Constructing appropriate models}

OOBNs vary in their detail, corresponding to stakeholders' objectives. We perceive that simplifying the process of using a management tool will increase the likelihood that it will be used. We present the ecology-decision-maker with two important tools that vary in their scope according to their audience. A detailed user-friendly OOBN corresponds to the needs of wildlife managers and owners to quantify the impact of specific management actions (Figure 3a). At the same time, abstract tool corresponds to the needs of government officials to rapidly asses structured risk in order to enable the user to visually conceptualize integrated variables at play decide and allocate resources efficiently (Figure $3 \mathrm{~b}$ ).

\section{Model predictions}

OOBNs present users with the need to consider wildebeest populations across South Africa as one metapopulation. The ratio between male black wildebeest and male blue wildebeest, coupled with complete barrier to gene flow as suggested by the biological $\mathrm{BN}$ and the spatial BN (respectively), were found to be the factor that most accurately predicted hybridization probabilities (Figure
3). This relationship may be attributed to the fact that hybridization mostly occurs as a result of mating encounters between blue wildebeest bulls and black wildebeest cows, and most management practices result in shifts in species abundance [5].

External and internal South African market forces are intertwined with EU meat export, translocation, eco-tourism, and hunting. These impact interlinked spatial and biological management decisions (Figure 3(a)). Implications of market shifts are quantified so that stakeholders can adapt management guidelines to counter them Figure 3(a). Decisions driven by market forces should aim to avoid unbalanced intraspecies population male ratios in order to prevent dramatic shifts in mating encounters.

\section{Conclusions}

\section{Maximizing 00BN utilization}

There is a need for a decision making tool that is flexible in its scope so that satisfactory probabilities of hybridization occurrences are defined by the model user, reflective of the objectives different stakeholders (Figure 4). We illustrate a pathway that leads to informed decision making (Figure 4).

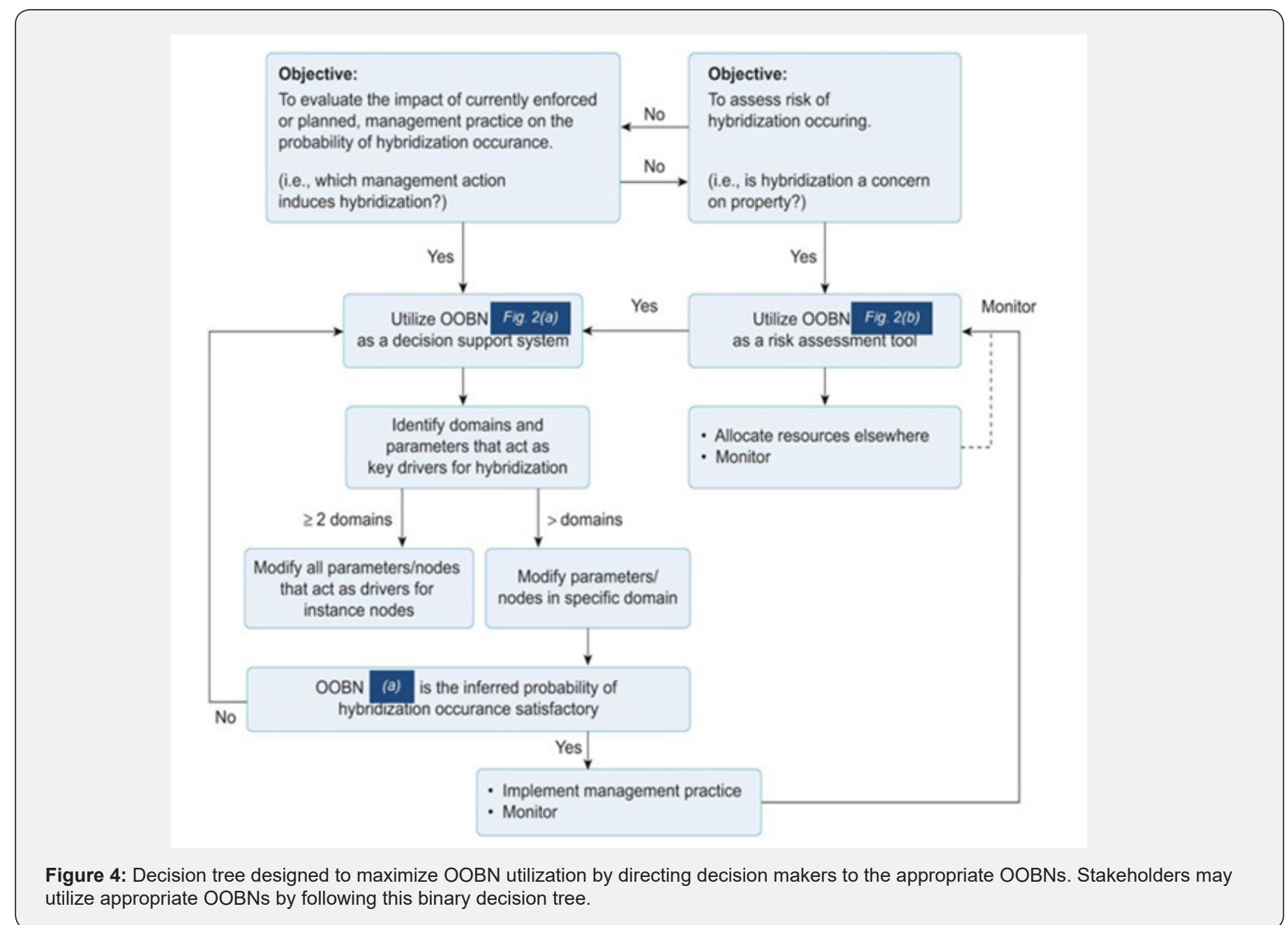

Tailoring OOBNs to the ecologist's decision-makers needs

By putting forth two OOBNs, which vary in their scope, we address the decision maker's needs. Governmental officials allocate significant expenditures in extreme cases. A primary example was the culling of entire populations in the case of the Abe Bailey Nature Reserve, where wildebeest were observed to be the product of varying degrees of introgression [12]. Given that 
the black wildebeest survived two bottlenecks [14], culling at such magnitudes is not only resource consuming, but may also have a negative effect on the regional metapopulation structure. In such instances, governmental ecologists may apply our proposed risk assessment tool to many private ranches, seeking to acquire permits for wildebeest translocations. Thereby maximizing manpower and associated costs.

The human thought process abstracts selectively from a hierarchy of class types [17]. Instance nodes are the linking points when working with different levels of abstraction. OOBN employ a similar process and is therefore relatable for the decision maker.

We illustrate that OOBNs provide a realistic tool for knowledge generators and users in which knowledge repository and retrieval takes place. Although the ecologist is confronted with scarce data and limited resources, expert knowledge may accurately provide a snap shot of the wicked problem at hand. We suggest that this form of knowledge engineering is what establishes BNs as optimal to solve for the ecologist's dilemma.

\section{Acknowledgments}

We seek to acknowledge the many South African conservation scientists, conservationists, and game stakeholders that willingly donated their time and information during workshops. We appreciate their willingness to participate. We additionally wish to thank peers whose insights have contributed to our final draft.

\section{Conflict of Interest}

No conflict of Interest.

\section{References}

1. Buchanan R (1992) Wicked problems in design thinking. Design issues 8(2): 5-21.

2. Jentoft S, Chuenpagdee R (2009) Fisheries and coastal governance as a wicked problem. Marine Policy 33(4): 553-560.

3. Benjamin-Fink N, Reilly BK 2017 (a) A road map for developing and applying object-oriented bayesian networks to "WICKED" problems. Eco Mod 360: 27-44.

4. Benjamin-Fink N, Reilly BK (2017(b)) Conservation implications of wildlife translocations; The state's ability to act as conservation units for wildebeest populations in South Africa. Globl Ecol and Cons 12: 46-58.

5. Allendorf FW, Leary RF, Spruell P, Wenburg JK (2001) The problems with hybrids: setting conservation guidelines. Trends Ecol Evol 16(11): 613622.

6. Barton NH, Hewitt GM (1985) Analysis of hybrid zones. Annl rev of Eco and Sys 16(1): 113-148.

7. Game ET, Meijaard E, Sheil D, McDonald-Madden E (2014) Conservation in a wicked complex world; challenges and solutions. Conserv Let 7(3): 271-277.

8. Mitroff II, Silvers A (2010) Dirty rotten strategies: How we trick ourselves and others into solving the wrong problems precisely. Stanford University Press, USA.
9. Hirsch PD, Adams WM, Brosius JP, Zia A, Bariola N, Dammert JL (2011) Acknowledging conservation trade-offs and embracing complexity. Conserv Biol 25: 259-264.

10. Boyd IL (2010) Assessing the effectiveness of conservation measures: Resolving the "“wicked"” problem of the Steller sea lion. Biol Conserv 143(7): 1664-1674.

11. Pearl J (2014) Probabilistic Reasoning in Intelligent Systems: Networks of Plausible Inference. Morgan Kaufmann Publishers, Inc. San Francisco, California write in the inference, USA.

12. Grobler JP, GB Hartl, N Grobler, A Kotze, K Botha, R Tiedemann (2005) The genetic status of an isolated black wildebeest (Connochaetes gnou) population from the Abe Bailey Nature Reserve, South Africa: microsatellite data on a putative past hybridization with blue wildebeest (C. taurinus). Mammalian Biology-Zeitschrift für Säugetierkunde 70(1): $35-45$.

13. Grobler JP, I Rushworth, JS Brink, P Bloomer, A Kotze, B Reilly, S Vrahimis (2011) Management of hybridization in an endemic species: decision making in the face of imperfect information in the case of the black wildebeest-Connochaetes gnou. Eur J of Wil Res 57(5): 997-1006.

14. Ackermann RR, JS Brink, S Vrahimis, B De Klerk (2010) Hybrid wildebeest (Artiodactyla: Bovidae) provide further evidence for shared signatures of admixture in mammalian crania. South African J of Sci 106(11/12): 1-4

15. Edmands S (2007) Between a rock and a hard place: evaluating the relative risks of inbreeding and outbreeding for conservation and management. Molecular ecology 16(3): 463-475.

16. Cai B, Zhao Y, Liu H, Xie M (2016) A Data-Driven Fault Diagnosis Methodology in Three-Phase Inverters for PMSM Drive Systems. IEEE Transactions on Power Electronics.

17. Weidl G, Madsen AL, Israelson S (2005) Applications of object-oriented Bayesian networks for condition monitoring, root cause analysis and decision support on operation of complex continuous processes. Comput Chem Eng 29(9): 1996-2009.

18. Wolfson LJ, Kadane JB, Small MJ (1996) Bayesian environmental policy decisions: two case studies. Ecol Appl: 1056-1066.

19. Düspohl M, Frank S, Döll P (2012) A review of Bayesian networks as a participatory modeling approach in support of sustainable environmental management. J of Sust Development 5(12): 1.

20. Marcot BG, Steventon JD, Sutherland GD, McCann RK (2006) Guidelines for developing and updating Bayesian belief networks applied to ecological modeling and conservation. Can J For Res 36(12): 3063-3074.

21. Pollino CA, Woodberry O, Nicholson A, Korb K, Hart BT (2007) Parameterisation and evaluation of a Bayesian network for use in an ecological risk assessment. Env Model Softw 22(8): 1140-1152.

22. Usitalo L (2007) Advantages and challenges of Bayesian networks in environmental modelling. Ecol Model 203(3): 312-318.

23. Norsys Software Corporation (2008) Netica. Version 4.08, Vancouver, Canada, URL: http://www.norsys.com/. Last accessed on December 10, 2016.

24. Spiegelhalter DJ (1993) Bayesian Analysis in Expert Systems. Stat Sci 8(3): 219-247.

25. Rhymer JM, D Simberloff (1996) Extinction by hybridization and introgression. Annual Review of Ecol and Sys: 83-109. 